\title{
dRDF: Entailment for Domain-Restricted RDF ${ }^{\star}$
}

\author{
Reinhard Pichler ${ }^{1}$, Axel Polleres ${ }^{2}$, Fang Wei ${ }^{1}$, and Stefan Woltran ${ }^{1}$ \\ ${ }^{1}$ Institut für Informationssysteme, Technische Universität Wien, Austria \\ \{pichler, wei, woltran\}@dbai.tuwien.ac.at \\ ${ }^{2}$ Digital Enterprise Research Institute (DERI), National University of Ireland, Galway \\ axel.polleres@deri.org
}

\begin{abstract}
We introduce domain-restricted RDF (dRDF) which allows to associate an RDF graph with a fixed, finite domain that interpretations for it may range over. We show that dRDF is a real extension of RDF and discuss impacts on the complexity of entailment in $\mathrm{dRDF}$. The entailment problem represents the key reasoning task for RDF and is well known to be NP-complete. Remarkably, we show that the restriction of domains in $\mathrm{dRDF}$ raises the complexity of entailment from NP- to $\Pi_{2}^{P}$-completeness. In order to lower complexity of entailment for both domain-restricted and unrestricted graphs, we take a closer look at the graph structure. For cases where the structure of RDF graphs is restricted via the concept of bounded treewidth, we prove that the entailment is tractable for unrestricted graphs and coNP-complete for domain-restricted graphs.
\end{abstract}

\section{Introduction}

The Resource Description Framework [18] provides means to publish and share metadata on the Web in a machine readable form. One of the features of RDF is to express incomplete metadata by so-called blank nodes, which allow to make statements about unknown resources, such as "I know somebody called 'Tim Berners-Lee' (but I don't know the URI identifying him)". In a sense, blank nodes can be viewed as existential variables in the data. In certain circumstances however, it is conceivable that more refined statements could be made about this "somebody". Normally, an RDF graph is interpreted over an infinite set of resources. However, one often has a concrete set of resources in mind when writing RDFs. For instance, we want to be able to say: "I don't know the URI identifying Tim, but I know that it is one of the URI's listed at: http: / /www . example.org/w3c-people', i.e. we want to assign blank nodes only to certain URI's from a restricted, finite set, but we just do not know which one.

In this paper, we introduce and investigate so-called domain-restricted $R D F(d R D F)$ graphs which allow to define exactly such restrictions. Domain-restricted RDF graphs are graphs for which interpretations are bound to a fixed, finite domain.

Example 1. The RDF graphs in Fig. 1 model collaboration links between various people. In the figure and subsequent examples, we use $:_{-} b_{1_{1_{-}}}: b_{2}, \ldots,,_{-}: b_{n}$ to denote blank nodes, quoted strings for literals of $L$, and colon separated pairs of alphanumeric strings

\footnotetext{
* The work of Axel Polleres has been supported by the European FP6 project inContext (IST034718) and by Science Foundation Ireland under the Lion project (SFI/02/CE1/I131).
}

S. Bechhofer et al.(Eds.): ESWC 2008, LNCS 5021, pp. 200-214. 2008.

(C) Springer-Verlag Berlin Heidelberg 2008 


\begin{tabular}{|c|c|c|}
\hline$G_{1}$ & $G_{2}$ & $G_{3}$ \\
\hline $\begin{array}{l}\left(\_: b_{1}, \text { foaf: name, "Fang"), }\right. \\
\left(\_: b_{2}, \text { foaf: name, "Stefan"), }\right. \\
\left(\_: b_{3}, \text { foaf: name, "Reini "), }\right. \\
\left(\_: b_{1},: \text { worksWith, }: b_{2}\right), \\
\left(\_: b_{2},: \text { worksWith, }: b_{3}\right)\end{array}$ & 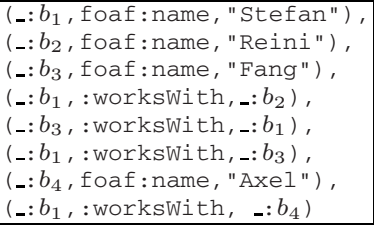 & $\begin{array}{l}\text { (_: } b_{2}, \text { foaf: name, "Stefan"), } \\
\left(\_: b_{1}, \text { foaf:name, "Axel"), }\right. \\
\left(\_: b_{2},: \text { worksWith, }: b_{1}\right)\end{array}$ \\
\hline
\end{tabular}

Fig. 1. Fictitious collaboration graphs published by Fang, Stefan W. and Stefan D

where the prefix may be empty for QNames/URIs 1 Graphs are sets of triples, as usual. The two fictitious graphs $G_{1}$ and $G_{2}$ describe metadata we assume to be published by two of the authors of this paper working at TU Vienna, Fang and Stefan. Fang's graph only talks about current employees of TU Vienna, Stefan's graph talks about current and past employees of TU Vienna, whereas $G_{3}$ denotes collaboration links of Stefan Decker, who talks in his graph only about current DERI employees. Even if we assume that lists of URIs to denote these domains 2 are published at some Web referenceable address, current RDF does not provide means to allow the respective publishers of the graphs $G_{1}-G_{3}$ to express or reference the domain they are talking about. dRDF fills exactly this gap.

The key reasoning task for RDF is deciding whether the information in one RDF graph is subsumed by what is said by another RDF graph - the RDF entailment problem. Entailment should intuitively be affected by restricting the domain of a graph. For instance, the graph $G_{3}$ is subsumed by $G_{2}$ modulo blank node renaming. Nevertheless, since these graphs talk about different domains, a reasoning engine aware of these domain restrictions should not conclude entailment here.

It is well known that blank nodes raise the complexity of the entailment problem to NP-completeness [14]. A major goal of this work is to search for realistic restrictions which might ensure tractability of the entailment problem. We thus study two kinds of restrictions: one is the restriction to a fixed, finite domain (i.e., dRDF) mentioned above. The other one is the restriction of the graph structure of the (RDF or $\mathrm{dRDF}$ ) graphs. More precisely, we investigate the entailment problem for graphs having bounded treewidth, which can be thought of as a generalization of acyclicity. It has been successfully applied to graph-related problems in many areas [38] where otherwise intractable problems have been proved to become tractable if the underlying graph structure has bounded treewidth.

One may expect that both kinds of restrictions decrease the complexity of the entailment problem. Somewhat surprisingly, we will show that the restriction to finite domains

\footnotetext{
${ }^{1}$ We use QNames in the sense of RDF notations such as Turtle [2], where e.g. foaf : name, : axel, or : worksWith stand for full URIs, but we leave out the actual namespace prefixes here, as they do not matter for illustration.

${ }^{2}$ Complete lists of URIs denoting all employees of TU Vienna, DERI, etc. should be easy to obtain. Institutes typically already do publish this data, see e.g. http://www.deri.ie/about/team/ or http://www.dbai.tuwien.ac.at/ staff/ It would be easy to write e.g. a GRDDL [9] transformation for those pages which creates lists of unique identifiers for their respective team members.
} 
does not help at all. In contrast, it even increases the complexity of entailment up to the second level of the polynomial complexity hierarchy, viz. to $\Pi_{2}^{P}$-completeness. On the other hand, we will show that the restriction to RDF graphs of bounded treewidth indeed makes the entailment problem tractable. We will present a polynomial-time algorithm for this case. Actually, also for dRDF graphs, bounded treewidth decreases the complexity of entailment by one level in the polynomial hierarchy; we thus end up with coNP-completeness rather than $\Pi_{2}^{P}$-completeness.

Our complexity results are summarized as follows. Note that the case of infinite resources and no restriction on the treewidth is well known to be NP-complete [14].

\begin{tabular}{|l|c|c|}
\hline & finite domain-restricted graphs & unrestricted graphs \\
\hline bounded treewidth & coNP-complete & in $\mathbf{P}$ \\
\hline unbounded treewidth & $\boldsymbol{\Pi}_{\mathbf{2}}^{\mathbf{P}}$-complete & NP-complete \\
\hline
\end{tabular}

The remainder of this paper is organized as follows. In Section 2 we will first review the formal definitions of RDF's syntax and semantics and introduce domain-restricted $R D F(d R D F)$ along the way. In this section we will also prove some important theoretical properties concerning general RDF entailment vs. dRDF entailment. The complexity of the entailment problem in case of domain-restricted RDF is dealt with in Section 3. The effect of bounded treewidth without or with domain-restriction is investigated in Section 4 and Section 5 respectively. We wrap up the paper with an outlook to related and future works and draw conclusions in Sections 6 and 7 . For an extended version of this paper including more detailed proofs we refer to [22].

\section{Preliminaries}

In this paper, we exclusively deal with simple RDF entailment, i.e., without giving any special semantics to the $\mathrm{RDF}(\mathrm{S})$ vocabulary. For short, we shall therefore use the term "RDF entailment" throughout this paper in order to refer to "simple RDF entailment". For the definition of the syntax and semantics of RDF graphs, we find the notation given in [14] more convenient than the one used for defining the standard semantics in [10]. It should be noted that for simple interpretations which we consider here both approaches are equivalent, apart from the fact that plain literals are ignored in [14]. It can be easily verified that our complexity results also hold if we stick literally to the definitions in [10].

\subsection{RDF Graphs and Domain-Restricted RDF Graphs}

We consider an infinite set $U$ (RDF URI references), an infinite set $B$ (blank nodes, also referred to as variables), and an infinite set $L$ (RDF literals). An $R D F$ triple is a triple of the form $\left(v_{1}, v_{2}, v_{3}\right) \in(U \cup B) \times U \times(U \cup B \cup L)$. In such a triple, $v_{1}$ is called the subject, $v_{2}$ the predicate, and $v_{3}$ the object. The union of the sets $U$ and $L$ is often denoted by $U L$, and likewise, $U \cup B \cup L$ is often denoted by $U B L$.

An $R D F$ graph (or simply a graph) is a set of RDF triples. A subgraph is a subset of a graph. The vocabulary of a graph $G$, denoted by $U L_{G}$, is the set of elements of $U L$ 
occurring in triples of $G$. A graph is ground if it has no blank nodes. RDF graphs are often represented as edge-labeled, directed graphs where a triple $(a, b, c)$ is represented by $a \stackrel{b}{\longrightarrow} c$.

A map is a function $\mu: U B L \rightarrow U B L$ preserving URIs and literals, i.e., $\mu(v)=v$ for all $v \in U L$. We define $\mu(G):=\{(\mu(s), \mu(p), \mu(o)) \mid(s, p, o) \in G\}$. A graph $G^{\prime}$ is an instance of $G$ if there exists a map $\mu$ with $G^{\prime}=\mu(G)$. With some slight ambiguity we say that there exists a map $\mu: G_{1} \rightarrow G_{2}$ if there is a map $\mu: U B L \rightarrow U B L$, such that $\mu\left(G_{1}\right)$ is a subgraph of $G_{2}$. Let $G_{1}$ and $G_{2}$ be graphs. The union $G_{1} \cup G_{2}$ is the set-theoretical union of their sets of triples.

Let $D \subseteq U L$ be a non-empty set of URI references and literals and $G$ be an RDF graph. A domain-restricted $R D F$ graph (dRDF graph) is a pair $\langle G, D\rangle$. Graphs such that $|D|=n$ is finite are also called finitely restricted (or simply restricted for short); graphs with $D=U L$ are also called unrestricted graphs. Sightly abusing notation, instead of $\langle G, U L\rangle$ we also write $G$ to denote unrestricted graphs.

\subsection{Semantics of (Domain-Restricted) RDF Graphs}

A simple interpretation $I=($ Res, Prop, Lit, $\varepsilon, I S, I L) \sqrt{3}$ of an RDF graph $G$ over vocabulary $U L_{G}$ is defined by (1) a non-empty set of resources Res (also called the domain of $I$ ) and of properties Prop, (2) a distinguished subset Lit $\subseteq$ Res, (3) an extension $\varepsilon(p r) \subseteq$ Res $\times$ Res for every property $p r \in$ Prop, and (4) mappings $I S: U_{G} \rightarrow$ Res $\cup$ Prop and $I L: L \rightarrow$ Lit.

We write $I($.$) to denote the valuation under the interpretation I$. We have $I(u):=$ $I S(u)$ for a URI $u$ and, $I(l):=I L(l)$ for a literal $l$. A triple $(s, p, o)$ has the value "true" in $I$ if $I S(p) \in$ Prop and $(I(s), I(o)) \in \varepsilon(I S(p))$; otherwise $(s, p, o)$ has the value "false". For a ground graph $G$, we have $I(G)=$ "true" if every triple of $G$ is true in $I$.

Blank nodes in non-ground graphs are interpreted as existentially quantified variables. Let $A: B \rightarrow$ Res be a blank node assignment (or an assignment, for short), and let $I$ be an interpretation. Then we write $[I+A]$ to denote the interpretation $I$ extended by the blank node assignment $A$. Clearly, $[I+A](b)=A(b)$ for blank nodes $b \in B$, while $[I+A](a)=I(a)$ for $a \in U L$. A non-ground graph $G$ is true in $I$, if there exists an assignment $A^{\prime}: B \rightarrow R e s$, s.t. every triple of $G$ is true in $\left[I+A^{\prime}\right]$. If a graph $G$ is true in an interpretation $I$, then we say that $I$ is a model of $G$ or $I$ satisfies $G$.

We say that an RDF graph $G_{1}$ entails the graph $G_{2}$, if every interpretation $I$ which satisfies $G_{1}$ also satisfies $G_{2}$. If this is the case, we write $G_{1} \models G_{2}$. This leads us to the RDF entailment problem: Given two RDF graphs $G_{1}, G_{2}$, does $G_{1} \models G_{2}$ hold? This problem is well known to be NP-complete [14]. We may assume w.l.o.g. that $U L_{G_{2}} \subseteq U L_{G_{1}}$, since otherwise $G_{1} \not \models G_{2}$ clearly holds (i.e., we can easily construct an interpretation $I$ which satisfies $G_{1}$ but not $G_{2}$ ).

Interpretations for a dRDF graph $\langle G, D\rangle$ restrict general RDF interpretations in the following sense. Given an interpretation $I=($ Res, Prop, Lit, $\varepsilon, I S, I L)$ and a set $D \subseteq U L$ we call the interpretation $I=\left(\right.$ Res $\cap D, \operatorname{Prop}$, Lit $\left.\cap D, \varepsilon, I S^{\prime}, I L^{\prime}\right)$ with $I S^{\prime}=I S_{\operatorname{Res} \cap D}$ and $I L^{\prime}=I L_{\text {Res } \cap D}$ the $D$-restriction of $I$, also written $I_{D}$. Note that

\footnotetext{
${ }^{3}$ As mentioned above, we are following the notation from [14]. Clearly, Res, Prop, Lit, $\varepsilon$, IS , and $I L$ correspond to $I R, I P, L V, I E X T, I S$, and $I L$, respectively, in [10].
} 
we do not restrict the domain of Prop in $I_{D}$. Since the purpose of domain-restrictions is mainly to restrict the values which blank nodes may take, we do not need to restrict properties - blank nodes are not allowed in property position in RDF anyway.

We define $d$-models as before with the only difference that for any interpretation $I$ its $D$-restriction is considered. I.e., given an interpretation $I$ and a dRDF graph $\langle G, D\rangle$, if $G$ is true in $I_{D}$, then we say that $I$ is a d-model of $\langle G, D\rangle$ or $I d$-satisfies $\langle G, D\rangle$.

Finally, we say that a dRDF graph $\left\langle G_{1}, D_{1}\right\rangle$ d-entails $\left\langle G_{2}, D_{2}\right\rangle$ (by overloading $=$ we write $\left.\left\langle G_{1}, D_{1}\right\rangle \models\left\langle G_{2}, D_{2}\right\rangle\right)$, if for any interpretation $I$ s.t. $I_{D_{1}}$ satisfies $G_{1}, I_{D_{2}}$ also satisfies $G_{2}$. Obviously, if $D_{1}$ contains an element not existing in $D_{2}$, then this condition can never be fulfilled. Indeed, if $c \in D_{1} \backslash D_{2}$, then we can easily construct a $D_{1}$-model of $G_{1}$ (where every URI in $G_{1}$ is mapped to $c$ ) which is not a $D_{2}$-model of $G_{2}$. Conversely, if $D_{2}$ contains elements not existing in $D_{1}$, then these elements play no role for d-entailment, i.e., we have $\left\langle G_{1}, D_{1}\right\rangle \models\left\langle G_{2}, D_{2}\right\rangle$ iff $\left\langle G_{1}, D_{1}\right\rangle \models\left\langle G_{2}, D_{1} \cap D_{2}\right\rangle$. Therefore, in the sequel, we shall restrict ourselves w.l.o.g. to the case $D_{1}=D_{2}$.

Example 2 (Example 1 cont'd). Getting back to the graphs in Fig. 1 it is easy to see that $G_{2} \models G_{3}$ and that $G_{1} \models G_{2}^{\prime}$, where $G_{2}^{\prime}$ is the graph obtained from $G_{2}$ by removing the last three statements of $G_{2}$. As mentioned earlier, Fang's graph $G_{1}$ talks only about people working at TU Vienna, i.e., it is restricted to the fixed domain $D_{1}=\{$ "Fang", "Stefan", "Reini" $\} \cup D_{T U V}$ where $D_{T U V}$ is a fixed, finite list of URIs which gives identifiers to all current TU Vienna employees and contains for instance the URIs : fangwei, : stefanwoltran, and :reinhardpichler. This list may be huge and instead of looking up all the real identifiers there, Fang still uses blank nodes as in the example for publishing her metadata. But in order to indicate the fact that her graph talks about a finite domain she publishes the dRDF graph $\left\langle G_{1}, D_{1}\right\rangle$. Likewise, Stefan publishes his collaboration links as graph $\left\langle G_{2}, D_{2}\right\rangle$. Stefan's graph is restricted to $D_{2}=D_{1} \cup D_{T U V o l d}$ where $D_{T U V \text { old }}$ is a finite list of identifiers of former TU Vienna members that also contains the URI : axelpolleres, for example. Both $\left\langle G_{1}, D_{1}\right\rangle \models\left\langle G_{2}^{\prime}, D_{2}\right\rangle$ and $\left\langle G_{2}, D_{2}\right\rangle \models G_{3}$ hold. However, $G_{3}$ is in fact none of the authors' but Stefan Decker's collaboration graph at DERI and restricted to the domain $D_{3}=\{$ "Stefan", "Axel " $\} \cup D_{D E R I}$ where $D_{D E R I}$ is the (again finite) list of identifiers of DERI employees that contains among others the URIs : axelpolleres and : stefandecker, but none of the other previously mentioned URIs. Obviously, $\left\langle G_{2}, D_{2}\right\rangle \not \models\left\langle G_{3}, D_{3}\right\rangle$ despite the fact $\left\langle G_{2}, D_{2}\right\rangle \models G_{3}$.

\subsection{Properties of (Domain-Restricted) Entailment}

Before we have a closer look at the complexity of this restricted form of the entailment problem, let us discuss some fundamental properties of (domain-restricted) entailment.

Proposition 1. Let $G_{1}, G_{2}$ be graphs and $D$ a finite domain. Then $G_{1} \models G_{2}$ implies $\left\langle G_{1}, D\right\rangle \models\left\langle G_{2}, D\right\rangle$ while the converse is, in general, not true.

Proof. Clearly, entailment implies d-entailment, since every d-model is also a model. To see that the converse is, in general, not true, consider the following counter-example: Let $G_{1}=\{(a, p, b),(a, p, c),(b, p, c)\}$ and $G_{2}=\{(x, p, x)\}$ where $a, b, c, p \in U$ and 
$x \in B$. Moreover, let $D=\left\{d_{1}, d_{2}\right\}$. Then $\left\langle G_{1}, D\right\rangle \models\left\langle G_{2}, D\right\rangle$ holds: Indeed, with $|D|=2$, any d-model $I$ of $\left\langle G_{1}, D\right\rangle$ assigns the same value $d_{i}$ (for some $i \in\{1,2\}$ ) to two URIs out of $\{a, b, c\}$. Hence, $G_{2}$ is true in $[I+A]$ with $A(x)=d_{i}$.

Proposition 2. Let $G_{1}, G_{2}$ be graphs and $D$ a finite domain with $|D| \geq\left|U L_{G_{1} \cup G_{2}}\right|$. Then $G_{1} \models G_{2}$ iff $\left\langle G_{1}, D\right\rangle \models\left\langle G_{2}, D\right\rangle$.

Proof. The "only if" direction immediately follows from Proposition 1 . The basic idea of the "if"-direction is that, for any interpretation, only the "active domain" (i.e, the elements in Res which are actually used for interpreting the elements in $U L_{G_{1} \cup G_{2}}$ ) is relevant. For details, see [22].

Intuitively, Proposition 2 states that entailment and d-entailment coincide for a sufficiently large domain $D$.

We conclude this section by showing that w.l.o.g. several simplified assumptions may be made, both for the entailment problem and the d-entailment problem.

A Skolemization of a graph $G$ is a ground instance of $G$ which maps every blank node in $G$ to some "fresh" URI reference. These fresh URI references are called the Skolem vocabulary. The Skolemization of $G$ is denoted as $s k(G)$. The usefulness of Skolemizations is due to the following property:

Lemma 1. Let $G_{1}, G_{2}$ be graphs and let sk $\left(G_{1}\right)$ be a Skolemization of $G_{1}$, s.t. the Skolem vocabulary is disjoint from both $G_{1}$ and $G_{2}$. Moreover, let $D$ be a finite domain. Then the following equivalences hold: $G_{1} \models G_{2} \Leftrightarrow \operatorname{sk}\left(G_{1}\right) \models G_{2}$ and $\left\langle G_{1}, D\right\rangle \models$ $\left\langle G_{2}, D\right\rangle \Leftrightarrow\left\langle s k\left(G_{1}\right), D\right\rangle \models\left\langle G_{2}, D\right\rangle$.

Proof. The correctness of this lemma in case of ordinary entailment is shown in [10]. The case of d-entailment can be shown by exactly the same arguments.

In other words, for both ordinary entailment and d-entailment, we may assume w.l.o.g. that the graph $G_{1}$ is ground. After having restricted the syntax, we show that also the set of models to be inspected by an (ordinary or d-) entailment test can be significantly restricted. In [10], entailment testing is reduced to Herbrand models. However, in case of domain-restricted graphs, we can of course not be sure that the Herbrand universe is contained in the finite domain $D$. We thus have to generalize the idea of Herbrand models to minimal models.

Definition 1. We call a model I of an RDF graph $G$ (resp. a dRDF graph $\langle G, D\rangle$ ) a minimal model of $G$ (resp. $\langle G, D\rangle$ ), if the extensions $\varepsilon(p r)$ in I are chosen minimal (for every $p r \in$ Prop) s.t. $G$ is true in I. In other words, for every property $p r \in$ Prop, a minimal model is characterized by the following relation

$$
\varepsilon(p r)=\left\{(I(s), I(o)) \mid(s, p, o) \in G_{1} \text { and } I S(p)=p r\right\} .
$$

Clearly, every Herbrand model is a minimal model while the converse is, in general, not true. The following lemma states that, for (d-) entailment testing, we may restrict ourselves to minimal models of $G_{1}$. 
Lemma 2. Let $G_{1}, G_{2}$ be graphs, s.t. $U L_{G_{2}} \subseteq U L_{G 1}$ and $G_{1}$ is ground. Moreover, let $D$ denote a finite domain. Then the following equivalences hold:

(a) $G_{1} \models G_{2}$ iff every minimal model I of $G_{1}$ satisfies $G_{2}$.

(b) $\left\langle G_{1}, D\right\rangle \models\left\langle G_{2}, D\right\rangle$ iff every minimal model I of $G_{1}$ with Res $\subseteq$ Datisfies $G_{2}$.

Proof. The restriction to minimal models of $G_{1}$ (resp. $\left\langle G_{1}, D\right\rangle$ ) is based on the following observation: Suppose that $G_{1}$ (or $\left\langle G_{1}, D\right\rangle$ ) is true in some interpretation $I$. It clearly remains true if we restrict $\varepsilon$ to $\varepsilon^{\prime}$ with $\varepsilon^{\prime}(p r)=\varepsilon(p r) \cap\{(I(s), I(o)) \mid(s, p, o) \in$ $G_{1}$ and $\left.I S(p)=p r\right\}$. In case (b), the restriction to interpretations $I$ with Res $\subseteq D$ is obvious since, in a d-interpretation, Res is restricted to a subset of $D$ anyway.

\section{Complexity of d-Entailment}

We are now ready to investigate the complexity of d-entailment testing. It turns out that it is one level higher in the polynomial hierarchy than without domain restrictions.

The $\Pi_{2}^{P}$ upper bound is easily established via the lemmas from Section 2.3 .

Lemma 3. The d-entailment problem is in $\Pi_{2}^{P}$.

Proof. Recall that, by Lemma 1, we may assume w.l.o.g. that $G_{1}$ is ground. Then the complementary problem " $\left\langle G_{1}, D\right\rangle$ does not d-entail $\left\langle G_{2}, D\right\rangle$ " can be decided by the following $\Sigma_{2}^{P}$-algorithm.

1. Guess an interpretation $I$ over the vocabulary of $G_{1} \cup G_{2}$, s.t. $G_{1}$ is true in $I$.

2. Check that for all assignments $A$ for the blank nodes in $G_{2}$, the graph $G_{2}$ is false in $[I+A]$. Clearly, this check can be done by a coNP-oracle.

The proof of the $\Pi_{2}^{P}$ lower bound is much more involved. Due to space limitations, we can only give a rough sketch here. For details, see [22]. The proof goes by a reduction from a restricted form of the so-called $H$-subsumption problem. H-subsumption was introduced in the area of automated deduction as a powerful technique of redundancy elimination (cf. [12]). Given two clauses $C, C^{\prime}$, and a Herbrand universe $H, C \leq_{s s}^{H} C^{\prime}$ holds, iff, for each substitution $\vartheta$ of the variables in $C^{\prime}$ to $H$, there exists a substitution $\mu$ of the variables in $C$ to $H$, such that $C \mu \subseteq C^{\prime} \vartheta$. In this paper we are only interested in the case that $H$ is a finite domain of constants. In [21], it was shown that the H-subsumption problem is $\Pi_{2}^{P}$-complete even if $C$ and $C^{\prime}$ consist of unnegated atoms only. However, we need a strongly restricted version of H-subsumption: In particular, we have to restrict the $\mathrm{H}$-subsumption problem to the setting, where no constants are allowed to occur in the clauses and where all predicates are binary. We call such problems total, binary $\mathrm{H}$-subsumption problems (TBH-subsumption, for short). Of course, it is a priori by no means clear that TBH-subsumption is still $\Pi_{2}^{P}$-hard. Hence, the $\Pi_{2}^{P}$-hardness proof essentially consists of two parts: the problem reduction from $\mathrm{TBH}$ subsumption to d-entailment and the $\Pi_{2}^{P}$-hardness proof of TBH-subsumption.

Lemma 4. The TBH-subsumption problem can be reduced in polynomial time to the $d$-entailment problem. 
Proof. Consider an instance $C \leq_{s s}^{H} C^{\prime}$ of the TBH-problem over some finite universe $H$. In $C, C^{\prime}$, all predicates are binary and all arguments of the atoms in $C$ and $C^{\prime}$ are first-order variables. W.l.o.g., the clauses $C$ and $C^{\prime}$ have no variables in common. Moreover, all predicates in $C$ also occur in $C^{\prime}$ (since otherwise $C \mathbb{Z}_{s s}^{H} C^{\prime}$ trivially holds) and all predicates in $C^{\prime}$ also occur in $C$ (since literals in $C$ with a predicate symbol not occurring in $C^{\prime}$ play no role at all in the H-subsumption test-they can never be matched by literals in $C$ ). We define the dRDF graphs $\left\langle G_{1}, D\right\rangle$ and $\left\langle G_{2}, D\right\rangle$ with $D=H, G_{1}=\left\{(s, p, o) \mid p(s, o) \in C^{\prime}\right\}$, and $G_{2}=\{(s, p, o) \mid p(s, o) \in C\}$, s.t. the vocabulary of $G_{1} \cup G_{2}$ is given as $U:=\left\{s, p, o \mid p(s, o) \in C^{\prime}\right\}$ and $L=\emptyset$. Moreover, we have $B=\{s, o \mid p(s, o) \in C\}$. In other words, $G_{1}$ is ground while $G_{2}$ contains only blank nodes. Clearly, this reduction is feasible in polynomial time. The correctness (i.e., $C \leq_{s s}^{H} C^{\prime} \Leftrightarrow\left\langle G_{1}, D\right\rangle \models\left\langle G_{2}, D\right\rangle$ ) is shown in [22].

Lemma 5. The TBH-subsumption problem is $\Pi_{2}^{P}$-hard.

Proof. The proof is highly involved and very technical. It proceeds in three steps: First, $\Pi_{2}^{P}$-hardness is shown for clauses using only ternary predicates over a 2-element Herbrand universe $H=\{a, b\}$. This result is then extended to an arbitrary, finite $H$ with $|H| \geq 2$. Finally, it is shown that $\Pi_{2}^{P}$-hardness still holds even if the clauses are built up from binary predicates only and $|H| \geq 4$; details are fully worked out in [22].

Putting the Lemmas 35 together, we immediately get the following result.

Theorem 1. The d-entailment problem is $\Pi_{2}^{P}$-complete.

In other words, the complexity of entailment increases from NP- to $\Pi_{2}^{P}$-completeness if we restrict the domain. This unexpected effect can be explained as follows. RDF entailment with unrestricted domain admits a syntactical characterization: $G_{1} \models G_{2}$ iff there exists a map from $G_{2}$ to $G_{1}$. The proof of the "only if" direction of this equivalence crucially depends on an argument via Herbrand interpretations of $G_{1}$ (see the interpolation lemma and its proof in [10]). Of course, this argument is no longer valid for dRDF-graphs if the domain $D$ is smaller than the Herbrand universe (note that the counter-example given in the proof of Proposition 1 is based on a similar idea).

\section{Efficient Entailment Through Bounded Treewidth}

In this section we first define the treewidth of an RDF graph, then show that the entailment problem of $G_{1} \models G_{2}$ can be solved in polynomial time, if $G_{2}$ has bounded treewidth. Recall that an RDF triple has the form $\left(v_{1}, v_{2}, v_{3}\right) \in(U \cup B) \times U \times(U \cup$ $B \cup L)$. Let us denote those triples $\left(v_{1}, v_{2}, v_{3}\right)$ where $v_{1}$ and $v_{3}$ are two distinct variables as blank triples. Moreover, we speak of semi-blank triples if only one of $v_{1}, v_{3}$ is a variable or if $v_{1}$ and $v_{3}$ are identical variables.

It is interesting to observe that the intractability of the RDF entailment problem $G_{1} \models G_{2}$ depends only on the blank triples in $G_{2}$. To see this, consider the ground and semi-blank triples in $G_{2}$ : finding a map of any ground triple is merely an existence test of the triple in $G_{1}$. Thus all the ground triples can be tested independently from each other. Now let us assume that $G_{2}$ contains only semi-blank triples with $k$ distinct 
variables. Assume further that $\left|G_{1}\right|=m$ and $\left|G_{2}\right|=n$. To test $G_{1} \models G_{2}$, we first partition all the triples of $G_{2}$ into $k$ disjoint sub-graphs $P_{1}, \ldots, P_{k}$, s.t. two triples belong to the same sub-graph if and only if they contain the same variable. For each $i$, let $n_{i}$ denote the cardinality $\left|P_{i}\right|$ of $P_{i}$. Clearly, $n_{1}+\cdots+n_{k}=n$. We can then check the entailment of the sub-graphs one by one. For each $P_{i(1 \leq i \leq k)}$, the variable in $P_{i}$ can be mapped to $m$ possible values. Because there is only one variable in $P_{i}$, for each map $\mu$, we have to execute the existence test $\mu\left(P_{i}\right) \subseteq G_{1}$, which takes maximum $m n_{i}$ steps. Thus in summary, the total cost of the entailment test is $\mathcal{O}\left(m^{2} n\right)$.

However, if the graph $G_{2}$ contains blank triples, it is possible that the variables are intertwined s.t. no variable can be tested independently, thus the number of possible maps is exponential in the size of the variables occurring in blank triples. Treewidth is a well-known metric on graphs that measures how tree-like a graph is. Many intractable problems become tractable, if the treewidth of the underlying structure is bounded.

We shall now show that the entailment problem $G_{1} \models G_{2}$ becomes tractable if the graph $G_{2}$ has bounded treewidth. Recall the syntactical characterization of entailment [10|14]: $G_{1} \models G_{2}$ iff there exists a map from $G_{2}$ to $G_{1}$. Hence, the entailment problem for unrestricted RDF graphs comes down to a special case of conjunctive query containment where all predicates are binary. Hence, the notion of treewidth and the tractability of conjunctive query containment in case of bounded treewidth (see e.g. [5]) naturally carry over to RDF graphs and the entailment problem. However, we prefer to give a native definition of tree decomposition for RDF graphs here, so that the RDF intuition is preserved. Likewise, we explicitly present an entailment algorithm in terms of the RDF terminology rather than by just referring to conjunctive queries.

We start by giving the definitions of tree decomposition and treewidth for an RDF graph. By the above considerations, we assume that the RDF graph does not contain any ground triple. We denote all the variables occurring in $G$ as $B_{G}$.

Definition 2. A tree decomposition $\mathcal{T}$ of an RDF graph $G$ is defined as $\left\langle T,\left(B_{i}\right)_{i \in T}\right\rangle$ where $T$ is a tree and each $B_{i}$ is a subset of $B_{G}$ with the following properties:

1. Every $b \in B_{G}$ is contained in some $B_{i}$.

2. For every blank triple $\left(v_{1}, v_{2}, v_{3}\right) \in G$, there exists an $i \in T$ with $\left\{v_{1}, v_{3}\right\} \subseteq B_{i}$.

3. For every $b \in B_{G}$, the set $\left\{i \mid b \in B_{i}\right\}$ induces a subtree of $T$.

The third condition is usually referred to as the connectedness condition. The sets $B_{i}$ are called the blocks of $\mathcal{T}$. The width of the tree decomposition $\left\langle T,\left(B_{i}\right)_{i \in T}\right\rangle$ is defined as $\max \left\{\left|B_{i}\right| \mid i \in T\right\}-1$. The treewidth of an RDF graph $G$ (denoted as $t w(G)$ ) is the minimal width of all tree decompositions of $G$. For a given $w \geq 1$, it can be decided in linear time whether some graph has treewidth $\leq w$. Moreover, in case of a positive answer, a tree decomposition of width $w$ can be computed in linear time [4].

Example 3. Consider the graph $G_{2}$ given in Fig. 2. The undirected graph and the tree decomposition are depicted in Fig. 3. The treewidth of $G_{2}$ is 2 .

Below, we describe an algorithm which, given the tree decomposition of $G_{2}$, tests $G_{1} \models G_{2}$ in polynomial time. The intuition behind the algorithm is as follows: we first construct partial maps from the nodes on the tree decomposition into $G_{1}$ (denoted 


\begin{tabular}{|c|c|}
\hline$G_{1}$ & $G_{2}$ \\
\hline 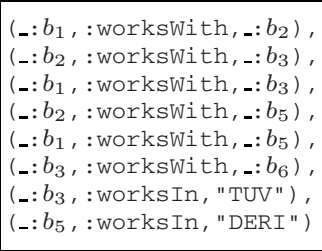 & 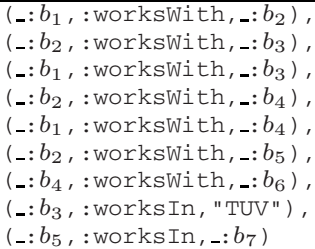 \\
\hline
\end{tabular}

Fig. 2. RDF graphs for Example 3
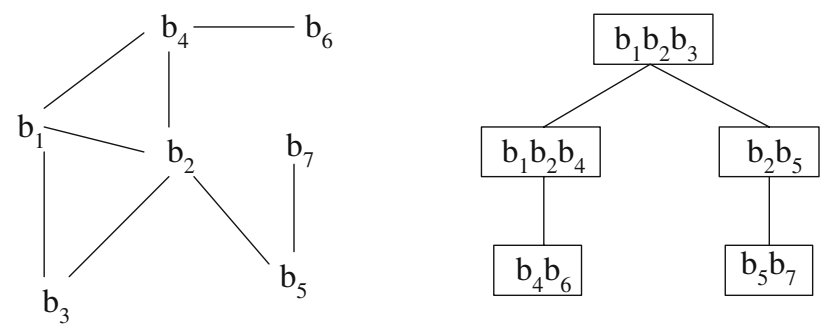

Fig. 3. Undirected graph of $G_{2}$ from Fig. 2 and the tree decomposition of $G_{2}$

as $M_{i}$ in the algorithm below), then successively merge those partial maps which are consistent with each other. If at last the merging succeeds, $G_{1} \models G_{2}$ holds, otherwise not. Note that the connectedness property of the tree decomposition allows us to merge such partial maps in a bottom up manner on the tree (by using the semi-join operation of relational algebra), in polynomial time. We thus carry over ideas proposed in [5] for testing conjunctive query containment to the RDF entailment problem.

Polynomial Time Algorithm. Let $\left\langle T,\left(B_{i}\right)_{i \in T}\right\rangle$ be the tree decomposition of the RDF graph $G_{2}$ with treewidth $k$. Given a node $i$ in $T, S_{i}$ is denoted as the union of all the blocks in the sub-tree rooted at $i$. The induced sub-graph $G\left[S_{i}\right]$ contains all the triples $\left(v_{1}, v_{2}, v_{3}\right)$ in $G_{2}$, such that either $v_{1}$ or $v_{3}$ belongs to $S_{i}$. We maintain for each node $i$ in $T$ a relation $M_{i}$. In the algorithm below, $\ltimes$ is the natural semi-join operator.

The Polycheck algorithm for checking $G_{1} \models G_{2}$ consists of the following steps:

1. For each node $i$ in $T$, generate the sub-graph $G_{i}^{\prime}$ which contains all the triples $\left(v_{1}, v_{2}, v_{3}\right)$ such that $\left\{v_{1}, v_{3}\right\} \subseteq B_{i} \cup U L_{G_{2}}$ and $\left\{v_{1}, v_{3}\right\} \cap B_{i} \neq \emptyset$.

2. Initialize the relation $M_{i}$ as follows: for each map $\mu$ from $G_{i}^{\prime}$ to $G_{1}$, the tuple $\mu\left(B_{i}\right)$ is in $M_{i}$.

3. Process the tree nodes bottom-up as follows: Suppose $i$ is a tree node in $T$ all of whose children have been processed. For each child $j$ of $i$, we set $M_{i}:=M_{i} \ltimes M_{j}$.

4. Let $r$ be the root of $T$. Then $G_{1} \models G_{2}$ if and only if $M_{r}$ is not empty.

Example 4. Let us continue with Example 3 With the given tree decomposition of $G_{2}$, we illustrate in Fig. 4 how the Polycheck algorithm works when testing $G_{1} \models G_{2}$.

Step 1: We need to generate the sub-graphs $G_{1}^{\prime}, \ldots, G_{5}^{\prime}$ for the nodes $1-5$ of the tree decomposition. For instance, $G_{4}^{\prime}$ is the sub-graph consisting of only one triple $\left(_{-}: b_{4}\right.$, : worksWith, _: $\left.b_{6}\right)$. 


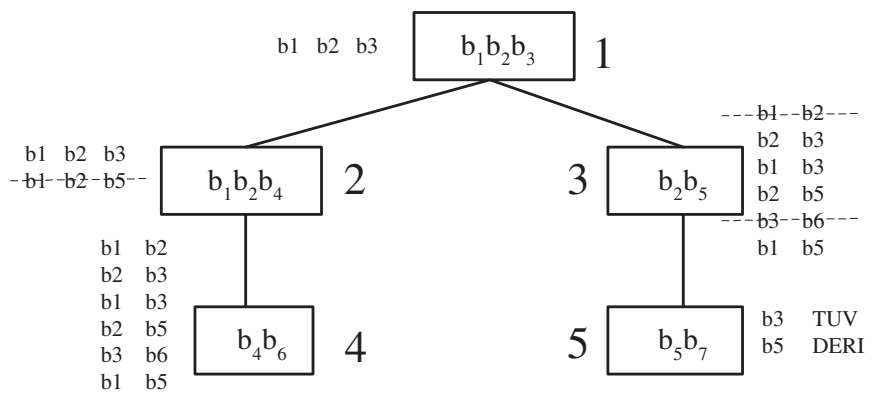

Fig. 4. Bottom up processing on the tree decomposition

Step 2: Next we generate the partial maps $M_{i(1 \leq i \leq 5)}$, which are given as the tables beside the tree nodes. Note that following the convention of relational databases, the variable names at each block give the relation schema for that block and every row of a table is called a tuple. For the time being, let us ignore the dotted lines drawn over the tuples. Now consider $M_{4}$. For every map $\mu$ with $\mu\left(\left(_{-}: b_{4},:\right.\right.$ worksWith, $\left.: b_{6}\right) \in G_{1}$, we insert the tuple $\mu\left({ }_{-}: b_{4},_{-}: b_{6}\right)$ into $M_{4}$. It is easy to verify that there are six distinct maps from $G_{4}^{\prime}$ to $G_{1}$, thus $M_{4}$ consists of six tuples.

Step 3: We execute semi-joins along the bottom-up traversal of the tree decomposition. The tables at the leaf nodes remain unchanged. Let us consider the semi-join operation $M_{2} \ltimes M_{4}$. By definition, the result of the semi-join is the set of those tuples $t$ in $M_{2}$ for which there is a tuple $t^{\prime}$ in $M_{4}$, s.t. $t$ and $t^{\prime}$ coincide on their common attributes (in our case $\left.b_{4}\right)$. Such a $t^{\prime}$ is called a partner of $t$. Now let us consider the first tuple $\left(b_{1}, b_{2}, b_{3}\right)$ in $M_{2}$. In this case, $b_{3}$ is the value for the common attribute $b_{4}$. A partner tuple $\left(b_{3}, b_{6}\right)$ in $M_{4}$ is found, thus the tuple $\left(b_{1}, b_{2}, b_{3}\right)$ remains in $M_{2}$. However, for the second tuple $\left(b_{1}, b_{2}, b_{5}\right)$, there does not exist any partner tuple in $M_{4}$. Therefore the tuple $\left(b_{1}, b_{2}, b_{5}\right)$ is deleted by the semi-join operation.

Step 4: Finally, the only tuple in $M_{1}$ remains after the semi-join operation with both $M_{2}$ and $M_{3}$, thus the entailment test succeeds.

Theorem 2. The algorithm Polycheck correctly decides whether $G_{1} \models G_{2}$.

Proof. We use induction on the number of nodes processed in the tree, with the following hypothesis: After node $i$ is processed, tuple $t \in M_{i}$ if and only if there is a map $\mu$ from $G\left[S_{i}\right]$ to $G_{1}$ such that $\mu\left(B_{i}\right)=t$. Thus when the root $r$ has been processed, $M_{r}$ is non-empty if and only if there is a map $\mu$ from $G\left[S_{r}\right]$ to $G_{1}$. Because $G\left[S_{r}\right]$ is $G_{2}$, we can therefore conclude that $G_{1} \models G_{2}$.

The induction hypothesis holds for the leaves, because of step 2, and the induced sub-graph $G\left[S_{l}\right]$ of any leaf node $l$ is the the graph $G_{l}^{\prime}$ we defined in the step 1 .

For the induction, assume that we have processed all the children $j_{1}, \ldots, j_{r}$ of node $i$. Suppose that $t \in M_{i}$ holds before the processing of node $i$. Let $\phi$ be the map of $G_{i}^{\prime}$ to $G_{1}$ s.t. $\phi\left(B_{i}\right)=t$. If $t \in M_{i}$ still holds after the processing of $i$ (i.e., the semi-join operations with all the child nodes), then for each $j_{k(1 \leq k \leq r)}$, there is a tuple $t_{k} \in M_{j_{k}}$, that agrees with $t$ on the variables in $B_{i} \cap B_{j_{k}}$. By the induction hypothesis, there is a map $\phi_{k}$ from $G\left[S_{j_{k}}\right]$ to $G_{1}$, such that $\phi_{k}\left(B_{j_{k}}\right)=t_{k}$. 
It remains to show that the maps $\phi, \phi_{1}, \ldots, \phi_{r}$ are consistent. Assume that $v$ occurs in the blocks $B_{j_{\alpha}}$ and $B_{j_{\beta}}$ of two children of $i$. According to the connectedness condition, $v$ occurs in $B_{i}$ too. Since $\phi, \phi_{j_{\alpha}}$ and $\phi_{j_{\beta}}$ agree on the common variables, $\phi, \phi_{j_{\alpha}}$ and $\phi_{j_{\beta}}$ are consistent. Let $\mu:=\phi \cup \phi_{1} \cup \ldots \cup \phi_{r}$. Then $\mu$ is clearly a map from $G\left[S_{i}\right]$ to $G_{1}$ such that $\mu\left(B_{i}\right)=t$.

Conversely, assume there is a map $\mu$ from $G\left[S_{i}\right]$ to $G_{1}$ such that $\mu\left(B_{i}\right)=t$, we show that $t$ is in $M_{i}$ after the processing of $i$. Clearly $t$ is in $M_{i}$ before the processing of $i$, because $G_{i}^{\prime}$ as defined in step 1 is a sub-graph of $G\left[S_{i}\right]$. Let $\phi_{1}, \ldots, \phi_{r}$ be the projection $\mu$ onto the variables in $S_{j_{1}}, \ldots, S_{j_{r}}$, and let $\phi$ be the projection of $\mu$ onto the variables in $B_{i}$. By the induction hypothesis, there is a tuple $t_{i} \in M_{j_{k}}$, where $1 \leq k \leq r$, such that $\phi_{k}\left(B_{j_{k}}\right)=t_{k}$. After step 2, there is a tuple $t \in M_{i}$, such that $\phi\left(B_{i}\right)=t$. Since $t$ agrees with the tuples $t_{1}, \ldots, t_{r}$ on all common attributes, $t$ is in $M_{i}$ after the processing of $i$.

Theorem 3. The entailment problem of $G_{1} \models G_{2}$ can be decided in polynomial time if $G_{2}$ has bounded treewidth.

Proof. Suppose that $\left|G_{1}\right|=n,\left|G_{2}\right|=m$ and $t w\left(G_{2}\right)=k-1$. Step (1): For each tree node $i$, we need to scan all the triples in $G_{2}$ to generate the subgraph $G_{i}^{\prime}$ of $G_{2}$. Since the size of the tree decomposition of $G_{2}$ is not more than $m$, we have an $m^{2}$ upper bound. Step (2): For block $B_{i}$ with size $k$, there are $n^{k}$ possible tuples to be checked. For each tuple $t$, we generate the map $\mu$ from $B_{i}$ to $t$. If $\mu\left(G_{i}\right) \subseteq G_{1}$, then $t$ is added to $M_{i}$. Thus, the cost for the initialization of all the nodes is $m n^{k}$. Step (3): Each semijoin operation of two $k$-ary relations takes $n^{2 k}$ (using primitive nested loops), thus the total cost is $m n^{2 k}$. In summary, we get the upper bound $\mathcal{O}\left(m^{2}+m n^{2 k}\right)$ on the time complexity of the algorithm Polycheck.

To summarize, the entailment problem $G_{1} \models G_{2}$ is intractable, only if the blank triples in $G_{2}$ are cyclic. We note that, in practice, an RDF graph contains rarely blank nodes, and even less blank triples. Hence, most of the real RDF graphs are acyclic or have low treewidth such as 2 , and the entailment can be tested efficiently with the above algorithm. For instance, all the graphs in Fig. 1 are acyclic and thus have $t w \leq 1$.

\section{Bounded Treewidth and d-Entailment}

In the previous section, we have seen for RDF graphs that bounded treewidth significantly decreases the complexity of entailment. We shall now prove a similar result for d-entailment, where bounded treewidth again has a positive impact on the complexity.

Lemma 6. The d-entailment problem of $\left\langle G_{1}, D\right\rangle \models\left\langle G_{2}, D\right\rangle$ is in coNP if $G_{2}$ has bounded treewidth.

Proof. Suppose that $t w\left(G_{2}\right)$ is bounded by some constant. Recall that, by Lemma 1 we may assume w.l.o.g. that $G_{1}$ is ground. Then the complementary problem of testing $G_{1} \not \forall G_{2}$ can be decided by the following NP-algorithm:

1. Guess an interpretation $I$ over the vocabulary of $G_{1} \cup G_{2}$, s.t. $G_{1}$ is true in $I$.

2. Check that there exists no assignments $A$ for the blank nodes in $G_{2}$, s.t. the graph $G_{2}$ is true in $[I+A]$. 
The check in step 2 comes down to an ordinary entailment test $G_{1}^{\prime} \not \forall G_{2}^{\prime}$ with $G_{1}^{\prime}:=$ $\left\{(I(s), I(p), I(o)) \mid(s, p, o) \in G_{1}\right\}$ and $G_{2}^{\prime}:=\left\{(I(s), I(p), I(o)) \mid(s, p, o) \in G_{2}\right\}$, where we stipulate $I(z)=z$ for the variables $z$ in $G_{2}$. We clearly, have $\operatorname{tw}\left(G_{2}\right)=$ $t w\left(G_{2}^{\prime}\right)$. Hence, by Theorem 3 , the check $G_{1}^{\prime} \not \models G_{2}^{\prime}$ is feasible in polynomial time.

Lemma 7. The d-entailment problem of $\left\langle G_{1}, D\right\rangle \models\left\langle G_{2}, D\right\rangle$ is coNP-hardfor bounded treewidth of $G_{2}$. It remains coNP-hard even if $t w\left(G_{2}\right)=0$ (i.e., the graph induced by the blank nodes consists of isolated nodes only).

Proof. We prove the coNP-hardness by reducing the well-known NP-complete problem of graph $\ell$-colorability with $\ell \geq 3$ to the complementary problem $\left\langle G_{1}, D\right\rangle \not \forall\left\langle G_{2}, D\right\rangle$.

Let $G=(V, E)$ be a graph with vertices $V$ and edges $E$. We define two RDF graphs $G_{1}$ and $G_{2}$ as $G_{1}:=\{(u, e, v) \mid(u, v)$ is an edge in $E\}$ and $G_{2}:=\{(x, e, x)\}$ for some blank node $x$. Clearly, $t w\left(G_{2}\right)=0$. Moreover, this reduction is feasible in polynomial time. It remains to show the correctness of this reduction, which can be seen as follows: By definition, $G$ is $\ell$-colorable iff there exists a mapping $\vartheta$, assigning different colors to any two adjacent vertices $u, v \in V$. Obviously, such an assignment exists iff there exists an interpretation $I$ sending all triples $(u, e, v)$ in $G_{1}$ to values $(I(u), I(e), I(v))$ with $I(u) \neq I(v)$. This, in turn, is the case iff there exists no blank node assignment $A$, s.t. $(A(x), A(x)) \in \varepsilon(I(e))$.

In summary, we thus have the following exact complexity classification.

Theorem 4. The d-entailment problem of $\left\langle G_{1}, D\right\rangle \models\left\langle G_{2}, D\right\rangle$ is coNP-complete if $G_{2}$ has bounded treewidth.

\section{Related and Future Work}

Our results touch upon many related issues on RDF reasoning and Semantic Web reasoning in general. First of all, we point out that the peculiarities of reasoning with open and restricted domains raised by $\mathrm{dRDF}$ are closely related to similar issues discussed in the context of reasoning with rules and ontologies [6].

Next, we have only discussed simple (d)RDF entailment in this paper. As for future works, it will be interesting to see, which implications restrictions on the domain will have when higher entailment regimes such as RDF entailment, RDFS entailment, D-entailment, or entailments in OWL variants are considered. Standard fragments of OWL are well-known to be syntactic variants of decidable Description Logics [1], i.e. OWL Light is reducible to SHIF(D) and OWL DL is reducible to SHOIN(D) [16]. We plan to investigate how (finite) domain-restrictions on the data affect the complexity of entailment in these languages, see also [1, Chapter 5]. Alternatively to finitely restricting the domain of interpretations for the whole graph it seems that restricting the blank nodes in an RDF graph to a finite, enumerated class (using OWL's oneOf constructor) could have similar effects, when we extend our considerations towards OWL. We are currently investigating respective generalizations of the definition of $\mathrm{dRDF}$ graphs. As for rule extensions on top of ontology languages, we remark that $\mathrm{RDF}(\mathrm{S})$ and some non-standard fragments of OWL entailment can be reduced to sets of 
Datalog rules [7/13]17/27] and thus combined straightforwardly with datalog rules on top. Note however, that subsumption of arbitrary Datalog programs is undecidable [26]. Issues get even more involved, when standard OWL fragments or (non-monotonic) rule languages are added on top of these languages (see [11]15|25] and references therein) since in the unrestricted case, even the satisfiability problem for rule-extended ontologies problem becomes undecidable. However, here domain-restrictions may turn out to be actually a good thing, since those cases become decidable for finite domains. A complete investigation of complexity classes for such combinations is on our agenda. In the context of (non-monotonic) rules extensions, let us mention that restricting the domain of interpretations is also related to restricting the scope of negation to closed sets of rules in non-monotonic rule languages for the Web, see [23] for further details.

As for related results on finding tractable fragments of RDF, Muñoz et al. [19] define a syntactic subclass of RDFS with $\mathcal{O}(n \log n)$ bounds for entailment (without blank nodes though), which our results complement.

Deciding whether a SPARQL [24] query has an answer is an extension of simple RDF entailment which is PSPACE complete in general but also NP-complete in many cases [20]. We expect that our results propagate to tractable fragments of SPARQL over unrestricted RDF as well as over dRDF graphs, which to define is on our agenda.

Bounded treewidth is a well-established method for identifying tractable subclasses of otherwise intractable problems. It has been successfully applied to a great variety of graph-related problems like network reliability, job scheduling, compiler optimization, model checking, etc. (see e.g., [3] $)$ ). To the best of our knowledge though, bounded treewidth has not yet been considered in the context of Semantic Web reasoning.

\section{Conclusions}

Entailment checking is the key reasoning task for RDF. In this work, we have investigated how the complexity of deciding entailment in RDF is affected by two restrictions. Firstly, we introduced dRDF, a variant of RDF which allows to associate an RDF graph with a fixed, finite domain that interpretations for it may range over. We have demonstrated that such restrictions are useful in environments where someone wants to make RDF statements over closed contexts such as enterprises or institutions. Secondly, we investigated restrictions of the graph structure of (d)RDF graphs. Particularly, we investigated the effect of restricting the structure of RDF graphs to bounded treewidth, which considerably lowered the complexity of entailment checking. As related works show, there are many promising directions for applying our results, such as finding further tractable algorithms for fragments of SPARQL, or applying respective restrictions beyond simple RDF entailment.

\section{References}

1. Baader, F., Calvanese, D., McGuinness, D.L., Nardi, D., Patel-Schneider, P.F. (eds.): The Description Logic Handbook. Cambridge (2003)

2. Beckett, D.: Turtle - Terse RDF Triple Language (November 2007), available at http: / / www.dajobe.org/2004/01/turtle/

3. Bodlaender, H.L.: A tourist guide through treewidth. Acta Cybern. 11(1-2), 1-22 (1993) 
4. Bodlaender, H.L.: A linear-time algorithm for finding tree-decompositions of small treewidth. SIAM J. Comput. 25(6), 1305-1317 (1996)

5. Chekuri, C., Rajaraman, A.: Conjunctive query containment revisited. Theor. Comput. Sci. 239(2), 211-229 (2000)

6. de Bruijn, J., Eiter, T., Polleres, A., Tompits, H.: On representational issues about combinations of classical theories with nonmonotonic rules. In: Lang, J., Lin, F., Wang, J. (eds.) KSEM 2006. LNCS (LNAI), vol. 4092, Springer, Heidelberg (2006)

7. de Bruijn, J., Heymans, S.: RDF and logic: Reasoning and extension. In: Proc. WebS 2007, IEEE Computer Society Press, Los Alamitos (2007)

8. Downey, R.G., Fellows, M.R.: Parameterized Complexity. Springer, Heidelberg (1999)

9. Conolly, D. (ed.): Gleaning Resource Descriptions from Dialects of Languages (GRDDL). W3C recommendation (September 2007)

10. Hayes, P. (ed.): RDF Semantics. W3C Recommendation (February 2004)

11. Eiter, T., Ianni, G., Polleres, A., Schindlauer, R., Tompits, H.: Reasoning with rules and ontologies. In: Reasoning Web 2006, Springer, Heidelberg (2006)

12. Fermüller, C.G., Leitsch, A.: Hyperresolution and automated model building. J. Log. Comput. 6(2), 173-203 (1996)

13. Grosof, B., Horrocks, I., Volz, R., Decker, S.: Description logic programs: Combining logic programs with description logic. In: Proc. WWW 2003, ACM Press, New York (2003)

14. Gutiérrez, C., Hurtado, C.A., Mendelzon, A.O.: Foundations of semantic web databases. In: Proc. PODS 2004, ACM Press, New York (2004)

15. Heymans, S., de Bruijn, J., Predoiu, L., Feier, C., Van Nieuwenborgh, D.: Guarded hybrid knowledge bases. Theory and Practice of Logic Programming (to appear, 2008)

16. Horrocks, I., Patel-Schneider, P.F.: Reducing OWL entailment to description logic satisfiability. In: Fensel, D., Sycara, K.P., Mylopoulos, J. (eds.) ISWC 2003. LNCS, vol. 2870, Springer, Heidelberg (2003)

17. Ianni, G., Martello, A., Panetta, C., Terracina, G.: Faithful and effective querying of RDF ontologies using DLV ${ }^{\mathrm{DB}}$. In: Proc. ASP 2007 (2007)

18. Klyne, G., Carroll, J.J. (eds.): Resource Description Framework (RDF): Concepts and Abstract Syntax. W3C Recommendation (February 2004)

19. Muñoz, S., Pérez, J., Gutiérrez, C.: Minimal deductive systems for RDF. In: Franconi, E., Kifer, M., May, W. (eds.) ESWC 2007. LNCS, vol. 4519, pp. 53-67. Springer, Heidelberg (2007)

20. Pérez, J., Arenas, M., Gutierrez, C.: Semantics and complexity of SPARQL. In: Cruz, I., Decker, S., Allemang, D., Preist, C., Schwabe, D., Mika, P., Uschold, M., Aroyo, L.M. (eds.) ISWC 2006. LNCS, vol. 4273, Springer, Heidelberg (2006)

21. Pichler, R.: On the complexity of H-subsumption. In: Gottlob, G., Grandjean, E., Seyr, K. (eds.) CSL 1998. LNCS, vol. 1584, Springer, Heidelberg (1999)

22. Pichler, R., Polleres, A., Wei, F., Woltran, S.: Entailment for domain-restricted RDF (ext.version). Tech. Report DBAI-TR-2008-59, available at http: / /www. dbai. tuwien.ac.at/research/report/dbai-tr-2008-59.pdf

23. Polleres, A., Feier, C., Harth, A.: Rules with contextually scoped negation. In: Sure, Y., Domingue, J. (eds.) ESWC 2006. LNCS, vol. 4011, Springer, Heidelberg (2006)

24. Prud'hommeaux, E., Seaborne, A. (eds.): SPARQL Query Language for RDF. W3C Proposed Recommendation (November 2007)

25. Rosati, R.: $\mathcal{D} \mathcal{L}+\log$ : Tight integration of description logics and disjunctive datalog. In: Proc. KR 2006, AAAI Press, Menlo Park (2006)

26. Shmueli, O.: Decidability and expressiveness aspects of logic queries. In: Proc. PODS 1987, ACM Press, New York (1987)

27. ter Horst, H.J.: Completeness, decidability and complexity of entailment for RDF Schema and a semantic extension involving the OWL vocabulary. JWS 3(2-3), 79-115 (2005) 